\title{
Extração de Métricas e Análise de Sentimentos em Comentários Web no Domínio de Hotéis
}

\author{
Roney L. de S. Santos ${ }^{1}$, Raimundo S. Moura ${ }^{1}$ \\ ${ }^{1}$ Departamento de Computação - Universidade Federal do Piauí (UFPI) \\ 64.049-550 - Teresina - PI - Brasil \\ roneylira@hotmail.com, rsmeufpi.edu.br
}

\begin{abstract}
The significant growth of the Web has made from it a rich source for the evaluate of public opinion on a specific entity. Consequently, the number of opinions available makes the decision-making process impossible if it was necessary to read and to analyse all reviews. This paper presents a Web application prototype where from a review are returned the feeling (positive, negative or neutral), its features and other analysis metrics using Natural Language Processing and Sentiment Analysis. Experiments show efficacy in the precision of reviews with negative polarity and recall of reviews with positive polarity in $84.93 \%$ and $94.33 \%$ respectively.
\end{abstract}

Resumo. O significativo crescimento da Web tem a tornado uma rica fonte para a avaliação da opinião pública sobre uma entidade específica. Consequentemente, o número de opiniões disponíveis torna impossível uma tomada de decisão se for necessário ler e analisar todas as opiniões. Este trabalho apresenta um protótipo de uma aplicação Web que a partir de um comentário seja retornado o sentimento (positivo, negativo ou neutro), suas características e outras métricas de análise utilizando técnicas de Processamento de Linguagem Natural e Análise de Sentimentos. Experimentos mostram eficácia nos resultados de precisão de comentários com polaridade negativa e cobertura de comentários positivos em $84.93 \%$ e $94.33 \%$ respectivamente.

\section{Introdução}

Quando um consumidor tem interesse por um produto ou serviço é comum que ele procure referências ou opiniões. Empresas que vendem produtos e disponibilizam serviços também são motivadas a ter conhecimento das opiniões dos consumidores, tendo que procurar formas de analisar essas informações para conduzir ações de marketing e tomada de decisão.

Pesquisadores da área de Processamento de Linguagen Natural (PLN) têm buscado extrair informações úteis de dados não estruturados. O termo big data se refere ao massivo conjunto de informações digitais coletadas. Todos os dias são criados 2.5 quinquilhões de bytes de dados, tanto que $90 \%$ dos dados do mundo hoje foram criados apenas nos últimos dois anos ${ }^{1}$. Essa grande quantidade de dados faz com que a análise manual se torne uma tarefa impossível, sendo necessária a criação de métodos automáticos para analisar os dados [Liu 2010].

\footnotetext{
${ }^{1}$ http://www.isaca.org/groups/professional-english/big-data/groupdocuments/big_data_top_ten_v1.pdf
} 
Dessa forma, para auxiliar no processo de análise e formação de opinião do consumidor, este trabalho propõe um protótipo de uma aplicação Web baseada no domínio de hotéis onde a entrada é um comentário e como saída são retornadas a polaridade do comentário (positivo, negativo ou neutro), as características encontradas no comentário, número de tuplas < característica, palavra opinativa $>$ e a corretude das palavras do comentário. Para construir as saídas da aplicação foram utilizadas técnicas de PLN e Análise de Sentimentos.

As saídas são métricas que serão utilizadas em sistemas para inferir os comentários mais úteis. Em alguns sites de hotéis, usuários podem votar em comentários que eles consideram úteis ou inúteis quando estão pesquisando sobre o hotel. Entretanto, nem sempre apenas informações de polaridade do comentário [Kim et al. 2006] são suficientes pois outros problemas, como por exemplo, comentários mais novos que nunca foram votados nunca serão lidos e votados [Li et al. 2013] podem acontecer.

Os experimentos mostraram que o protótipo tem grande cobertura dos comentários positivos e uma boa precisão dos comentários negativos. Os comentários considerados neutros podem ter sua polaridade alterada caso uma análise de contexto fosse realizada. $\mathrm{O}$ protótipo da aplicação também mostrou as características encontradas e suas polaridades, para uma análise mais fiel do usuário de hotéis.

O restante do artigo está organizado como: A Seção 2 relata a Fundamentação Teórica deste trabalho. A Seção 3 destaca os trabalhos relacionados. A Seção 4 discorre sobre o funcionamento do protótipo da aplicação Web. A Seção 5 mostra os experimentos realizados e os resultados. Por fim, a Seção 6 conclui o artigo e discute os trabalhos futuros.

\section{Análise de Sentimentos e Mineração de Opinião}

Análise de Sentimentos ou Mineração de Opinião é o estudo computacional de opiniões, avaliações, atitudes e emoções das pessoas referenciando entidades, indivíduos, questões, eventos, tópicos e seus atributos. O estudo é realizado sobre a emoção do usuário em relação ao item, classificando a emoção em positiva, negativa ou neutra, sendo determinada por técnicas de PLN. É tecnicamente desafiador e muito útil.

[Liu 2012] define uma opinião sendo uma quíntupla $\left(e_{i}, a_{i j}, s_{i j k l}, h_{k}, t_{l}\right)$ onde:

- $e_{i}$ é o nome de uma entidade;

- $a_{i j}$ é um aspecto de uma entidade (opcional);

- $s_{i j k l}$ é o sentimento sobre um aspecto $a_{i j}$ da entidade $e_{i}$, emitido pela pessoa $h_{k}$ no tempo $t_{l}$;

- $h_{k}$ é o detentor da opinião (opinion holder);

- $t_{l}$ é o instante no qual a opinião foi expressada por $h_{k}$.

Um típico sistema de Análise de Sentimentos envolve três etapas: i) extração de opiniões e suas características; ii) identificação da orientação semântica da opinião; e iii) sumarização dos resultados. A detecção do sentimento de um texto pode ocorrer em diferentes níveis: documento, sentença e entidade ou aspecto. Pesquisas que contém mineração de opinião e métricas para sumarização da informação são encontradas em [Khan et al. 2009], [Seerat and Azam 2012] e [Vinodhini and Chandrasekaran 2012]. 


\section{Trabalhos Relacionados}

Nesta seção são discutidos os conceitos utilizados neste artigo através de trabalhos relacionados, como aplicações Web usando Análise de Sentimentos e utilização de ontologias no âmbito do PLN.

São vários os estudos onde a extração de sentimento de características em comentários de consumidores é realizada através de Mineração de Opinião abrangendo técnicas de PLN [Li et al. 2009, Hu and Liu 2004, Htay and Lynn 2013]. [Aravindan and Ekbal 2014] propõem técnicas baseadas em mineração de dados e aprendizado de máquina para analisar comentários automaticamente, extraindo as características do produto e determinando a polaridade de cada característica. Seus experimentos mostraram que o classificador teve uma precisão de $79.67 \%$ quando aplicados a cinco produtos populares.

[Farina et al. 2014] introduziram um framework onde permite aos desenvolvedores a executar um classificador de sentimentos e a validação dos resultados tanto em um ambiente local quanto em um ambiente distribuído (web), permitindo seleções de comentários em tempo real e representação gráfica de várias dimensões dos comentários (tempo, local, palavras-chave, fontes e o sentimento).

Redes Sociais também têm a atenção dos pesquisadores quando se trata de Análise de Sentimentos. [Sarlan et al. 2014] desenvolveram uma protótipo de uma aplicação web que através de uma vasta quantidade de tweet $^{2}$ é possível classificar as perspectivas do dos consumidores através do sentimento extraído da mensagem textual.

A utilização de aprendizado de máquina é comum na análise do sentimento de um comentário. Trabalhos utilizando Máquinas de Vetores de Suporte (Support Vector Machine, SVM) [Mullen and Collier 2004, Tan et al. 2011], Sistemas Fuzzy [de Sousa et al. 2015, Santos et al. 2016, Indhuja and Reghu 2014, Dragoni et al. 2014] e Redes Neurais Artificiais (RNA) [Sharma and Dey 2012a, Sharma and Dey 2012b] são amplos na área. [Laryea et al. 2015] utilizaram um aprendizado de máquina supervisionado para classificar comentários da Amazon em cinco tipos: positivo, negativo, conselho, neutro e sem sentimento. Segundo eles, alguns comentários contém soluções técnicas, maneiras improvisadas de resolver os problemas, frustrações, felicidade, entre outros.

Para trabalhos onde existe um domínio especifíco, ontologias são desenvolvidas para facilitar a análise e extração de características. [De Freitas and Vieira 2013] apresentam métodos de avaliação para identificar polaridade em comentários escritos em Português de acordo com características descritas na ontologia definida. [Yannis and Evanthia 2012] propõem uma aplicação que constrói uma base de ontologia automaticamente através de textos de resultados de mecanismos de pesquisa com suas respectivas regras semânticas. No âmbito de ontologias referentes a hotéis, [Hasany and Selamat 2011] apresentam um sistema que coleta informações de hoteis através de ontologia e linguagem natural para tomada de decisão. Experimentos mostraram eficácia na ajuda da aplicação na tomada de decisão do usuário.

Neste trabalho o foco está além do sentimento do comentário. Métricas que são importantes para uma futura utilidade do comentário em outros contextos são coletadas e

\footnotetext{
${ }^{2}$ Mensagens de texto da rede social Twitter
} 
disponibilizadas para o usuário através de uma aplicação web. Além disso, nenhum dos trabalhos relacionados citados nesta seção analisam as características individualmente, conhecendo a polaridade de cada uma, o que é uma das propostas desse trabalho.

\section{Protótipo}

O protótipo da aplicação Web recebe um comentário como entrada e retorna como saídas: i) a polaridade do comentário (positivo, negativo ou neutro); ii) as características e suas polaridades, analisando as palavras opinativas referentes; iii) a quantidade de tuplas $<$ característica, palavra opinativa $>$ encontradas; e iv) a corretude das palavras no comentário. As etapas de execução são mostradas na Figura 1.

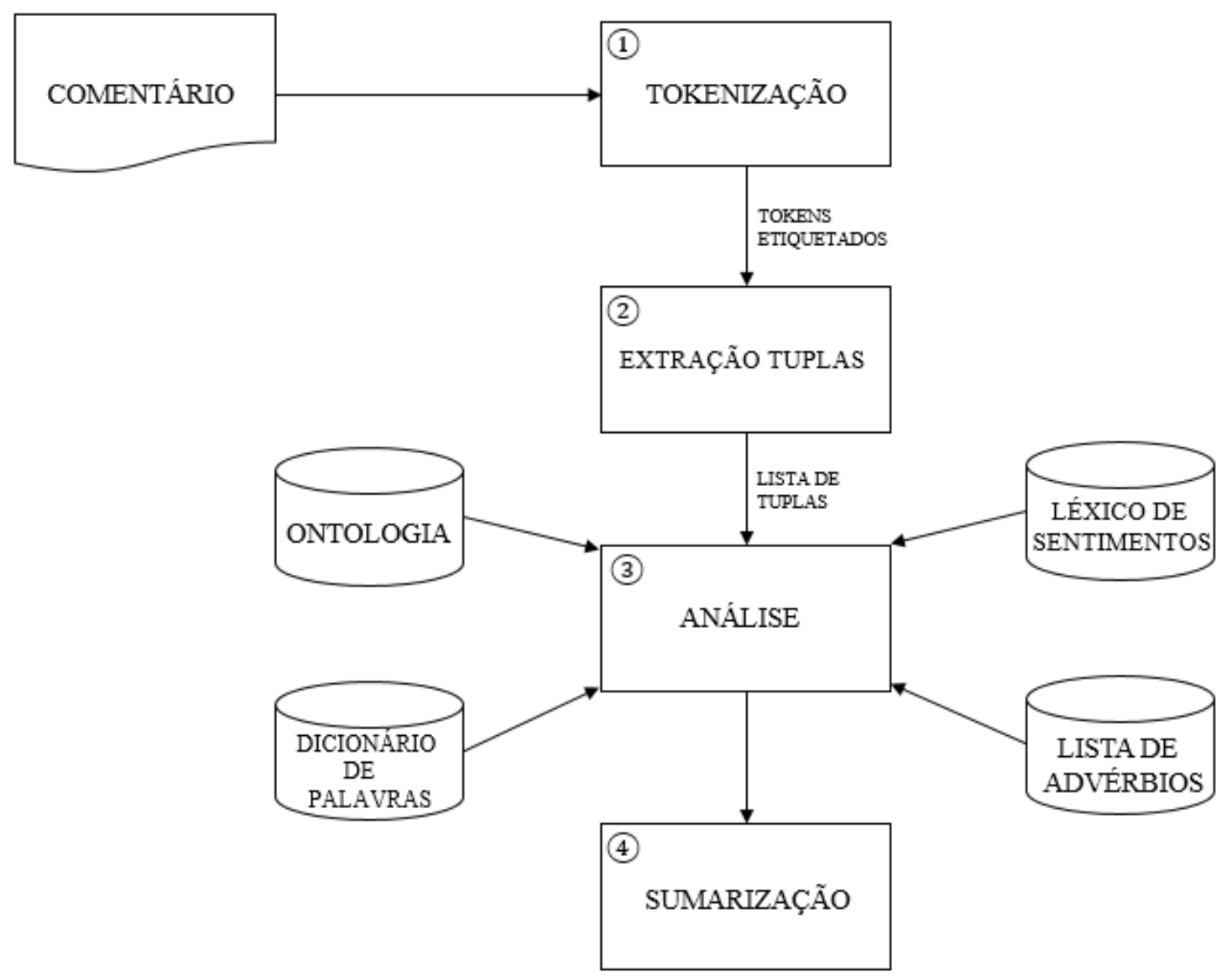

Figura 1. Protótipo: etapas de execução

Dado um comentário, a primeira etapa do protótipo foi realizar a separação das palavras do comentário em tokens (tokenização). Antes dos tokens serem submetidos ao etiquetador, é realizado um pré-processamento que substitui as preposições "da", "do", "das" e "dos" em uma estrutura "preposição + artigo", por exemplo, da = de+a. Esse procedimento foi necessário pois o etiquetador não reconhece essas contrações como preposições e consequentemente não reconheceria palavras compostas como "café da manhã".

A segunda etapa do protótipo consistiu em extrair as tuplas (palavra, classe gramatical) do comentário. Uma lista com tuplas foi gerada como resultado do etiquetador. 
As classes gramaticais foram definidas pelo etiquetador Mac-Morpho presente no NLTK (Natural Language Toolkit) [Bird et al. 2012].

Logo após, na terceira etapa, ocorreu a análise do comentário. Para a realização dessa análise, foram construídas quatro bases de dados. Inicialmente, para a construção das bases, foram coletados 2.216 comentários do site Booking. $\mathrm{com}^{3}$ de um hotel escolhido aleatoriamente. A coleta foi feita utilizando um Web crawler construído com a ferramenta Scrapy que captura os comentários através do arquivo HTML da página. Foram coletados a data, o autor, a nota e o comentário em geral.

Esses comentários serviram de base para uma análise manual. Essa análise manual foi executada coletando 30 comentários aleatoriamente da base de comentários e identificando características, palavras opinativas, advérbios e verbos presentes nos comentários. Em alguns comentários foi possível identificar os sintagmas (nominal, verbal e preposicional). Foram identificados vários padrões linguísticos que foram compactados em cinco padrões principais, descritos na Figura 2. O padrão 1 coleta as características e palavras opinativas presentes no comentário, contendo ou não advérbios antes do adjetivo. Caso exista um verbo de ligação entre a característica e a palavra opinativa, o padrão 1 também reconhece a estrutura "sujeito + verbo de ligação + predicativo do sujeito". O padrão 2 coleta as características e um ou mais adjetivos que se referenciam à ela. $\mathrm{O}$ padrão 3 por sua vez coleta as características e suas palavras opinativas quando aparecem no comentário na ordem inversa. O padrão 4 verifica se junto das características se encontram advérbios e verbos, uma vez que alguns verbos também transmitem sentimentos que podem ser explorados [Klavans and Kan 1998]. Por fim, o padrão 5 verifica o inverso do padrão 4, já que em alguns casos o verbo pode vir antes do advérbio.

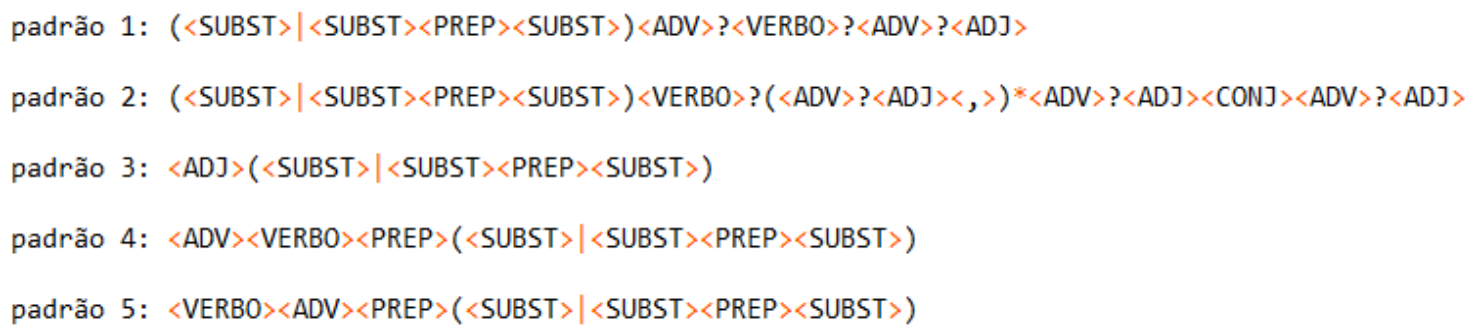

Figura 2. Padrões linguísticos identificados

Com o uso das características extraídas na análise manual, foi criada uma ontologia com as principais características de hotéis encontradas. O Booking.com define 7 características em cada página de cada hotel. Da análise manual foram encontradas mais 51 características, totalizando 58. Com o intuito de aumentar o escopo da ontologia, foi utilizado o ConceptNet [Liu and Singh 2004] coletando os sinônimos das características previamente encontradas. Foram encontradas mais 28 características, totalizando 86 características na base. Uma filtragem manual foi realizada a fim de eliminar as características com o mesmo stemming ${ }^{4}$, finalizando com 81 características. Essa ontologia força a aplicação a ser direcionada ao domínio de hotéis e está disponível online ${ }^{5}$.

\footnotetext{
${ }^{3}$ http://www.booking.com

${ }^{4}$ Termo usado na linguística para descrever o processo de redução (flexionado ou derivado) da palavra.

${ }^{5}$ https://goo.gl/HuKuGY
} 
A fim de fazer uma análise mais profunda do sentimento, foi construída uma lista de advérbios, sugerida inicialmente por [de Sousa et al. 2015], contendo 76 advérbios, a qual engloba boa parte dos advérbios da língua portuguesa e suas polaridades. Proveniente da análise manual realizada anteriormente, 6 advérbios coletados foram adicionados na base de advérbios, totalizando 82 advérbios.

Para analisar o sentimento de cada comentário foi utilizado o Sentilex-PT [Silva et al. 2012]. Uma base foi construída extraindo as palavras do arquivo disponibilizado pelo Sentilex-PT. Dentre os atributos de cada palavra, apenas a polaridade da palavra foi considerada. Para a realização da tarefa de corretude das palavras do comentário, foi construido um dicionário de palavras. A base de palavras foi retirada da WordNet [Dias-Da-Silva 2010] e contém 250.196 palavras.

Com todas as bases construídas, a aplicação foi capaz de analisar a polaridade de cada característica, a quantidade de tuplas e a corretude de cada comentário. A partir do momento que um comentário é submetido para análise, a aplicação utiliza todas as bases construídas, uma vez que a aplicação segue o domínio de hotéis.

Tomando como base os padrões encontrados (ver Figura 2), a lista de tuplas foi analisada para cada padrão a fim de salvar quais tuplas contém características, adjetivos, advérbios e verbos. A aplicação retorna o número de tuplas encontradas no comentário.

Ao mesmo tempo a polaridade do comentário foi analisada. Os adjetivos e verbos foram submetidos ao Sentilex-PT que retornou um número inteiro referente à sua polaridade: 1 para positivo, -1 para negativo ou 0 para neutro. Caso junto ao adjetivo contenha um advérbio, a polaridade do adjetivo é alterada após o advérbio ser submetido à base de advérbios. Por exemplo, se o adjetivo "bom" estiver acompanhado do advérbio "muito", sua polaridade é aumentada para 1,5. Por outro lado, se o adjetivo "ruim" estiver acompanhado do mesmo advérbio, sua polaridade é diminuída para -1,5. Ao final, as polaridades de cada adjetivo ou do conjunto advérbio+adjetivo do comentário são somadas e caso a soma resulte em um valor maior que 0 o comentário é considerado positivo; caso resulte em valor menor que 0 o comentário é considerado negativo e por fim, caso resulte em $0 \mathrm{o}$ comentário é considerado neutro.

Por fim foram extraídos todos os adjetivos referentes a cada característica para analisar a impressão geral do comentário sobre cada uma delas. Essa informação é importante devido ao fato de que o usuário pode pesquisar apenas por algumas características do hotel, como quartos e limpeza, em vez do hotel em geral. Essa análise se deu pela ontologia construída. Para cada característica do comentário presente na base de características, os adjetivos (e advérbios quando o acompanhando) eram salvos para a análise de polaridade. Essa análise seguia as mesmas regras da polaridade do comentário, verificando a polaridade do adjetivo pelo Sentilex-PT, dos advérbios pela base de advérbios, somando os valores e resultando na polaridade daquela característica.

Ao final de todo o processo, na quarta etapa, a aplicação mostra na tela de resultados a polaridade do comentário, as características encontradas no comentário, um gráfico informando quais são as características positivas, negativas e neutras, a quantidade de tuplas encontradas e a corretude do comentário. A Figura 3 mostra a tela de resultados da aplicação. 


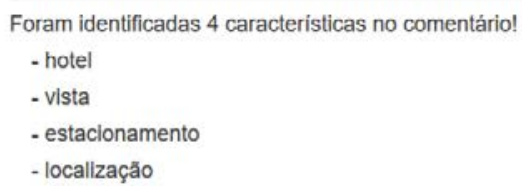

Figura 3. Protótipo: tela de resultados

\section{Experimentos e Resultados}

Para a validação do protótipo da aplicação $W e b$ foi realizado um experimento que verifica a precisão e cobertura (recall) da polaridade dos comentários analisados pelo protótipo, sendo mostrados os resultados obtidos.

A realização do experimento contou com uma base de 328 comentários aleatoriamente coletados da base existente, construída na etapa de análise. O número de comentários foi escolhido através de uma análise estatística que leva em consideração o nível de confiança e a margem de erro da amostra. Para esse trabalho o nível de confiança escolhido foi de $95 \%$ e a margem de erro escolhida foi de $5 \%$.

Os 328 comentários foram analisados manualmente por três especialistas da área de linguística, graduandos em Letras Português no mesmo nível de curso, definindo cada comentário como positivo, negativo ou neutro. Primeiramente foi verificada a análise dos dois primeiros especialistas, onde se houvesse conflito de escolha da polaridade, a análise do terceiro especialista definiria a polaridade para o comentário.

Após a conclusão da análise manual, os comentários foram submetidos ao protótipo da aplicação Web que retornou, entre outras saídas, a polaridade do comentário. Dos 328 comentários submetidos, o protótipo retornou 212 comentários positivos, 73 comentários negativos e 43 comentários neutros. Em comparação com os resultados da análise manual, houve aumento de $25 \%$ na detecção dos comentários positivos e queda de $34 \%$ e $26 \%$ na detecção dos comentários negativos e neutros, respectivamente.

O aumento na detecção dos comentários positivos e a queda na detecção dos comentários negativos estão relacionados devido ao modo com que o protótipo define a polaridade do comentário, somando as polaridades das palavras opinativas referentes a 
cada característica encontrada. Em alguns comentários de contexto negativo continham tuplas com palavras opinativas de polaridade positiva, tendo como consequência o incremento positivo na polaridade final do comentário e transformando um comentário de contexto negativo em positivo. Por exemplo, a Figura 4 mostra um comentário de contexto negativo que o protótipo identificou como positivo. Tuplas com polaridade positivas (suíte ótima, vista maravilhosa) são maioria em comparação com tuplas com polaridade negativas (equipe, meio lenta, serviço, meio devagar).

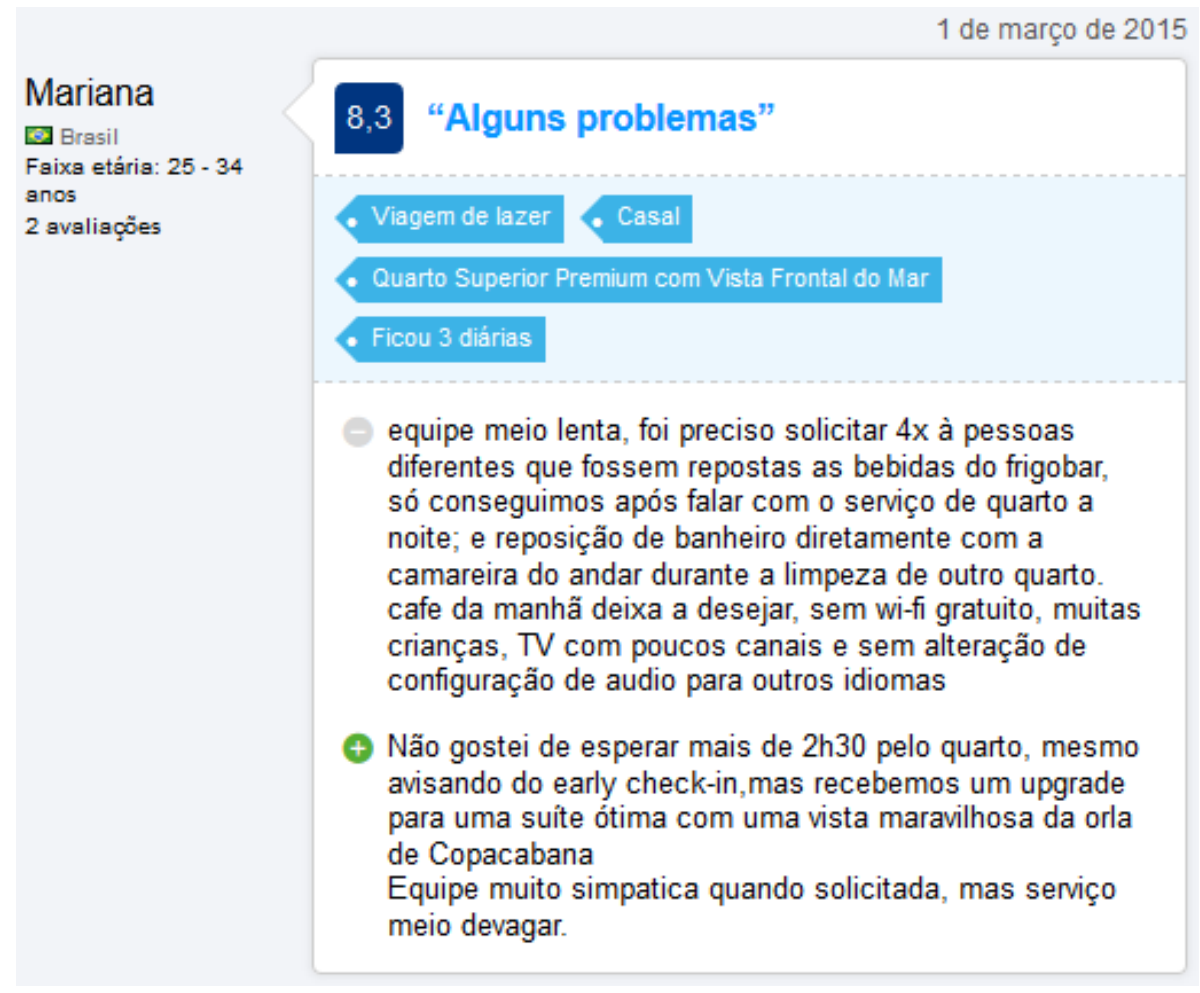

Figura 4. Comentário do Booking.com

Utilizando os resultados da execução de todos os comentários, foi feita a análise da polaridade dos comentários verificando a precisão, cobertura (recall) e medida-f de cada. Tomando como base a análise manual, os comentários foram separados por polaridade e comparados com os resultados do protótipo, que também foram separados da mesma maneira. A Tabela 1 mostra os resultados.

Tabela 1. Precisão, Recall e Medida-F de cada polaridade

\begin{tabular}{|c|c|c|c|}
\cline { 2 - 4 } \multicolumn{1}{c|}{} & Precisão & Recall & Medida-F \\
\hline Positivos & $70.75 \%$ & $94.33 \%$ & $80.86 \%$ \\
\hline Negativos & $84.93 \%$ & $55.85 \%$ & $67.39 \%$ \\
\hline Neutros & $55.81 \%$ & $41.37 \%$ & $47.52 \%$ \\
\hline
\end{tabular}

Exemplificando para melhor entendimento: o experimento de precisão verificou o quão preciso foi o protótipo, conseguindo acertar os comentários que são realmente positivos pela análise dos especialistas. O protótipo encontrou 212 comentários positivos mas apenas 150 foram considerados positivos pela análise manual, alcançando assim a pre- 
cisão de $70.75 \%(150 / 212=0.7075)$ dos comentários positivos. O experimento de cobertura (recall) verificou quantos foram os comentários positivos encontrados pelo protótipo que são realmentes positivos de acordo com a análise manual. A análise manual descreveu 159 comentarios como positivos enquanto o protótipo encontrou 150 comentários positivos, cobrindo então $94.33 \%$ dos comentários $(150 / 159=0.9433)$. A medida-F é uma média harmônica da precisão e cobertura recall. O cálculo da medida-F é realizado segundo a equação

$$
\frac{2 * p * r}{p+r}
$$

onde $p$ representa o valor da precisão e $r$ representa o valor da cobertura (recall).

A partir dos resultados do experimentos verificou-se uma grande cobertura de comentários positivos e uma boa precisão de comentários negativos. Os comentários considerados neutros pelo protótipo podem ter a polaridade alterada para positivo ou negativo analisando o contexto do comentário.

\section{Conclusão}

Este trabalho apresentou o protótipo de uma aplicação Web baseada no domínio de hotéis que analisa os comentários e retorna como resultado informações como a polaridade do comentário, quantidade de tuplas <característica, palavra opinativa $>$ a corretude das palavras escritas no comentário e a polaridade das características encontradas no comentário.

Observando a polaridade dos comentários, os resultados foram satisfatórios. Com bons números na precisão e recall tanto de comentários com polaridade positiva quanto negativa, pode-se considerar que os padrões linguísticos utilizados na detecção das tuplas $<$ característica, palavra opinativa $>$ são corretos. Porém, em alguns comentários de contexto negativo as tuplas encontradas eram de polaridade positiva, o que de acordo com o método utilizado para definir a polaridade do comentário, a polaridade era invertida. A utilização do contexto pelo ConceptNet é um dos trabalhos futuros planejados.

$\mathrm{Na}$ análise manual foi observado que alguns comentários poderiam ter uma melhor análise se houvesse um dicionário de gírias, neologismos, erros mecânicos e contrações. Em relação às contrações, um método de pré-processamento foi implementado para o protótipo se adaptar ao etiquetador das palavras. Um outro trabalho futuro seria a construção do dicionário que englobaria os outros problemas citados anteriormente, já que eles não dependem do domínio inserido do comentário.

A corretude do comentário também pode ser melhorada. Em [Torruella and Capsada 2013] são citadas algumas medidas de análise da riqueza léxica do texto, verificando entre outras características, o tamanho do texto em diferentes tipos de escrita.

A fim de incrementar o protótipo da aplicação Web, algumas funcionalidades futuramente serão implementadas. Uma delas é a condição do usuário ter uma opção de submeter um conjunto de comentários e a aplicação Web mostrar os resultados em geral, separando as polaridades dos comentários e das características encontradas no conjunto de comentários para melhor análise. 
Uma outra funcionalidade a ser implementada no futuro é a importância do comentário. A ideia é baseada em [de Sousa et al. 2015] que utiliza Sistema Fuzzy com três entradas: reputação do autor, quantidade de tuplas e corretude do comentário. A diferença para o trabalho futuro é que uma outra métrica será utilizada: o tempo que o comentário foi postado, uma vez que em relação ao domínio de hotéis, o tempo é importante para o usuário tomar sua decisão. Além disso, as entradas serão ponderadas com o que o usuário mais se identifica e os comentários serão sumarizados em ordem de importância de acordo com as métricas utilizadas.

\section{Referências}

Aravindan, S. and Ekbal, A. (2014). Feature extraction and opinion mining in online product reviews. pages 94-99.

Bird, S., Klein, E., and Loper, E. (2012). Natural Language Processing with Python. O`Reilly.

De Freitas, L. and Vieira, R. (2013). Ontology-based feature level opinion mining for portuguese reviews. pages 367-370.

de Sousa, R. F., Rabelo, R. A. L., and Moura, R. S. (2015). A fuzzy system-based approach to estimate the importance of online customer reviews. In 2015 IEEE International Conference on Fuzzy Systems (FUZZ-IEEE), pages 1-8.

Dias-Da-Silva, B. C. (2010). Brazilian portuguese wordnet: A computational linguistic exercise of encoding bilingual relational lexicons. International Journal of Computational Linguistics and Applications, 1(1-2):137-150.

Dragoni, M., Tettamanzi, A. G. B., and Costa Pereira, C. (2014). Semantic Web Evaluation Challenge: SemWebEval 2014 at ESWC 2014, Anissaras, Crete, Greece, May 25-29, 2014, Revised Selected Papers, chapter A Fuzzy System for Concept-Level Sentiment Analysis, pages 21-27. Springer International Publishing.

Farina, J., Mazuran, M., and Quintarelli, E. (2014). New Trends in Databases and Information Systems: 17th East European Conference on Advances in Databases and Information Systems, chapter Extraction, Sentiment Analysis and Visualization of Massive Public Messages. Springer International Publishing.

Hasany, N. and Selamat, M. (2011). Answering user queries from hotel ontology for decision making. pages 123-127.

Htay, S. S. and Lynn, K. T. (2013). Extracting product features and opinion words using pattern knowledge in customer reviews. The Scientific World Journal, 2013(394758).

$\mathrm{Hu}, \mathrm{M}$. and Liu, B. (2004). Mining opinion features in customer reviews. In Proceedings of the 19th National Conference on Artifical Intelligence, pages 755-760.

Indhuja, K. and Reghu, R. P. C. (2014). Fuzzy logic based sentiment analysis of product review documents. In Computational Systems and Communications (ICCSC), 2014 First International Conference on, pages 18-22.

Khan, K., Baharudin, B., Khan, A., and e Malik, F. (2009). Mining opinion from text documents: A survey. In 3rd IEEE International Conference on Digital Ecosystems and Technologies, pages 217-222. 
Kim, S.-M., Pantel, P., Chklovski, T., and Pennacchiotti, M. (2006). Automatically assessing review helpfulness. In Proceedings of the 2006 Conference on Empirical Methods in Natural Language Processing, pages 423-430.

Klavans, J. and Kan, M.-Y. (1998). Role of verbs in document analysis. In Proceedings of the 36th Annual Meeting of the Association for Computational Linguistics and 17th International Conference on Computational Linguistics - Volume 1, ACL '98, pages 680-686.

Laryea, B., Choi, C.-H., Jung, I.-S., Lee, K.-H., and Cho, W.-S. (2015). Web application for sentiment analysis using supervised machine learning. International Journal of Software Engineering and its Applications, 9(1):191-200.

Li, M., Huang, L., Tan, C.-h., and Wei, K. K. (2013). Helpfulness of online product reviews as seen by consumers : source and content features. International journal of electronic commerce : IJEC, 17(4):101-136.

Li, Z., Zhang, M., Ma, S., Zhou, B., and Sun, Y. (2009). Information Retrieval Technology: 5th Asia Information Retrieval Symposium, AIRS 2009, Sapporo, Japan, October 21-23, 2009. Proceedings, chapter Automatic Extraction for Product Feature Words from Comments on the Web, pages 112-123. Springer Berlin Heidelberg.

Liu, B. (2010). Handbook of natural language processing, chapter Sentiment analysis and subjectivity. Chapman and Hall/CRC.

Liu, B. (2012). Sentiment analysis and opinion mining. Morgan and Claypool.

Liu, H. and Singh, P. (2004). Conceptnet \&mdash; a practical commonsense reasoning tool-kit. BT Technology Journal, 22(4):211-226.

Mullen, T. and Collier, N. (2004). Sentiment analysis using support vector machines with diverse information sources. In Proceedings of EMNLP 2004, pages 412-418.

Santos, R. L. S., Rabelo, R. A. L., and Moura, R. S. (2016). An experimental study based on fuzzy systems and artificial neural networks to estimate the importance of reviews about product and services. In 2016 International Joint Conference on Neural Networks (IJCNN 2016), page "to appear".

Sarlan, A., Nadam, C., and Basri, S. (2014). Twitter sentiment analysis. pages 212-216.

Seerat, B. and Azam, F. (2012). Opinion mining: Issues and challenges (a survey). International Journal of Computer Applications, 49(9):42-51.

Sharma, A. and Dey, S. (2012a). An artificial neural network based approach for sentiment analysis of opinionated text. In Proceedings of the 2012 ACM Research in Applied Computation Symposium, pages 37-42.

Sharma, A. and Dey, S. (2012b). A document-level sentiment analysis approach using artificial neural network and sentiment lexicons. SIGAPP Appl. Comput. Rev., 12(4):6775 .

Silva, M. J., Carvalho, P., and Sarmento, L. (2012). Building a sentiment lexicon for social judgement mining. In Proceedings of the 10th International Conference on Computational Processing of the Portuguese Language, pages 218-228. 
Tan, C., Lee, L., Tang, J., Jiang, L., Zhou, M., and Li, P. (2011). User-level sentiment analysis incorporating social networks. In Proceedings of the 17th ACM SIGKDD International Conference on Knowledge Discovery and Data Mining, pages 13971405 .

Torruella, J. and Capsada, R. (2013). Lexical statistics and tipological structures: A measure of lexical richness. Procedia - Social and Behavioral Sciences, 95:447 - 454.

Vinodhini, G. and Chandrasekaran, R. (2012). Sentiment analysis and opinion mining: a survey. International Journal of Advanced Research in Computer Science and Software Engneering, 2(6).

Yannis, P. and Evanthia, K. (2012). An ontology-based question answering system exploiting search engines' results. pages 426-429. 